\title{
Frequent Genetic Mismatch between Vaccine Strains and Circulating Seasonal Influenza Viruses, Hong Kong, China, 1996-2012
}

\author{
Martin C.W. Chan, Maggie H. Wang, Zigui Chen, David S.C. Hui, Angela K. Kwok, \\ Apple C.M. Yeung, Kun M. Liu, Yun Kit Yeoh, Nelson Lee, ${ }^{1}$ Paul K.S. Chan
}

The World Health Organization selects influenza vaccine compositions biannually to cater to peaks in temperate regions. In tropical and subtropical regions, where influenza seasonality varies and epidemics can occur yearround, the choice of vaccine remains uncertain. Our 17year molecular epidemiologic survey showed that most influenza $A(H 3 N 2)(9 / 11)$ and $B(6 / 7)$ vaccine strains had circulated in East Asia $>1$ year before inclusion into vaccines. Northern Hemisphere vaccine strains and circulating strains in East Asia were closely matched in 7 (20.6\%) of 34 seasons for H3N2 and 5 (14.7\%) of 34 seasons for B. Southern Hemisphere vaccines also had a low probability of matching (H3N2, 14.7\%; B, 11.1\%). Strain drift among seasons was common (H3N2, 41.2\%; B, 35.3\%), and biannual vaccination strategy (Northern Hemisphere vaccines in November followed by Southern Hemisphere vaccines in May) did not improve matching. East Asia is an important contributor to influenza surveillance but often has mismatch between vaccine and contemporarily circulating strains.

$\mathrm{I}$ nfluenza is a major cause of illness and death worldwide. The human influenza virus spreads rapidly (average reproduction number of 1.28 ), typically infects $5 \%-10 \%$ of the population during seasonal epidemics, and results in 3-5 million cases of severe acute lower respiratory tract infection and $\approx 500,000$ deaths per year globally $(1,2)$. Continuous evolution of the single-stranded influenza virus results in antigenic drift of its surface proteins hemagglutinin (HA) and neuraminidase $(3,4)$. Antigenic drift occurs on average 2-8 years in response to host immune selection pressure and is most frequently seen in influenza $\mathrm{A}(\mathrm{H} 3 \mathrm{~N} 2)$, followed by influenza B and influenza A(H1N1). Antigenic

Author affiliation: The Chinese University of Hong Kong, Hong Kong, China

DOI: https://doi.org/10.3201/eid2410.180652 drift confers the virus an ability to escape immunity induced by previous infection or immunization (5).

Effectiveness of current influenza vaccines is predominantly determined by matching between vaccines and circulating strains (6). To achieve the best possible vaccine matching, the World Health Organization (WHO) has changed its recommended vaccine composition 12 times for influenza $\mathrm{A}(\mathrm{H} 3 \mathrm{~N} 2), 10$ times for influenza $\mathrm{B}$, and 6 times for influenza $A(H 1 N 1)$ since 1998 (7). WHO reviews influenza vaccine composition each February and September to provide timely recommendation for temperate regions of the Northern and Southern Hemispheres, respectively. Tropical and subtropical regions are expected to choose 1 of the 2 recommended vaccine compositions. However, influenza seasonality varies more in the tropics and subtropics, where epidemics can occur year-round. Moreover, a national policy on the choice and timing of vaccination is lacking in many tropical and subtropical countries, where $60 \%$ of the world's population resides ( 8 ).

We analyzed the 2 most frequently drifting influenza types, A(H3N2) and B, in Hong Kong during 1996-2012 to examine matching between vaccine strains and circulating field viruses to document the challenges faced in this region. We evaluated options on choosing the Northern or Southern Hemisphere vaccine compositions and the strategy of an annual or biannual vaccination.

\section{Methods}

\section{Study Samples}

We conducted a retrospective molecular epidemiologic study to analyze the HA sequences of influenza A(H3N2) and influenza $\mathrm{B}$ viruses that circulated during a 17-year period (1996-2012) in Hong Kong, a subtropical city in

${ }^{1}$ Current affiliation: University of Alberta, Edmonton, Alberta, Canada. 
East Asia $\left(22^{\circ} 17^{\prime} 7.87^{\prime \prime} \mathrm{N}, 114^{\circ} 9^{\prime} 27.68^{\prime \prime} \mathrm{E}\right)$ with a high population density of 57,250 persons $/ \mathrm{km}^{2}$ in the most densely populated district, Kwun Tong (9). Hong Kong is an international hub. As recorded in 2011, Hong Kong received $>34$ million visitors from neighboring regions, including South and Southeast Asia, Taiwan, mainland China, and Macao, and an average of $\approx 650,000$ cross-boundary trips between Hong Kong and mainland China were recorded daily $(10,11)$. In this study, we used Hong Kong as a proxy to reflect the challenges faced in East Asia.

We focused on influenza $\mathrm{A}(\mathrm{H} 3 \mathrm{~N} 2)$ and influenza $\mathrm{B}$, rather than $\mathrm{A}(\mathrm{H} 1 \mathrm{~N} 1)$, because changes in vaccine composition were more frequent for the former 2 viruses. Clinical specimens were derived from patients admitted to the Prince of Wales Hospital, which serves 9\% (0.6 million) of Hong Kong's population (12). We sequenced an average of 30 influenza $\mathrm{A}(\mathrm{H} 3 \mathrm{~N} 2)$ and 30 influenza $B$ clinical samples per year, except for 2009, when influenza A(H1N1)pdm09 predominated. Selection was prioritized for samples collected during different months to reveal the whole annual spectrum of circulating viruses.

\section{Identifying Closest Vaccine Strains and Matched Seasons}

We identified vaccine strain closest to each circulating virus on the basis of HA sequence analysis. We extracted viral RNA from specimens using the PureLink Viral RNA/ DNA Mini kit (Thermo Fisher, Waltham, MA, USA). The full-length influenza $\mathrm{A}(\mathrm{H} 3 \mathrm{~N} 2) \mathrm{HA}$ encoding region was amplified and Sanger sequenced according to our previously described method (13). Similarly, the full-length influenza B HA encoding region was amplified with primers FLUB-UNI9-HAF1 (5'-AGC AGA AGC AGA GCA TTT TYT AAT ATC-3') and FLUB-HAR1 (5'-ACA AGC AAA CAA GYA CYA CAA YAA AG-3') and Sanger sequenced. We downloaded ancestral influenza $\mathrm{A}(\mathrm{H} 3 \mathrm{~N} 2)$ and influenza B HA sequences from GenBank. Information about WHO-recommended vaccine strains and sequences were retrieved from the Influenza Research Database (14). When the recommendation included alternative vaccine strains, each of them was included for analysis.

We conducted neighbor-joining phylogenetic inference on the basis of the HA sequences using MEGA6 (15). Resulting trees were visualized using FigTree version 1.4.2. We then matched each circulating strain to a closest influenza $\mathrm{A}(\mathrm{H} 3 \mathrm{~N} 2)$ or influenza $\mathrm{B}$ vaccine strain based on pairwise protein sequence distances as calculated by the Poisson model (16). Circulating strains with equal distance to 2 vaccine strains were assigned to the vaccine recommended in an earlier year. Strains with distances $>2$ times the SD from the mean distance of all strains were regarded as outliers and thus had no closest vaccine strain assigned.
When the circulating viruses and their closest vaccine strain corresponded in time, the season was regarded as matched. On the other hand, when the circulating viruses coincided with vaccine strains from a previous season (e.g., circulating strain from 2012 matching vaccine strain from 2010), the season was regarded as not matched. This matching scheme was based on the rationale that WHO changes the influenza vaccine composition only when substantial antigenic drift has occurred, and suboptimal effectiveness would be expected for the previous vaccine compositions.

\section{Seasonality and Protection Period}

Unlike temperate regions, peak seasons of influenza in the subtropics are not restricted to winter months. Because previous studies have revealed a biannual pattern of influenza activity in Hong Kong and surrounding regions $(12,17)$, we divided the surveyed 17-year period into 34 influenza seasons. We grouped calendar months under winter and summer influenza seasons on the basis of the average monthly number of hospital admissions of patients for whom influenza was laboratory confirmed that were recorded during the study period.

The Hong Kong Government Influenza Vaccination Programme recommends use of the Northern Hemisphere influenza vaccines and begins annual vaccination every November. Therefore, we defined the protection period of Northern Hemisphere vaccines as from November through October of the following year. Although Southern Hemisphere vaccines are not typically used in Hong Kong, for this analysis we regarded the protection period of these vaccines to be from May through April of the following year.

\section{Results}

During the 17-year study period, 8,011 persons with influenza A and 2,079 with influenza B were admitted to the Prince of Wales Hospital. Both infections exhibited 2 seasonal peaks in most years (Figure 1). Most influenza admissions occurred from late winter through early spring (late January through early March) and around summer (MaySeptember), although the summer peak of influenza B varied more and was less prominent than that of influenza A. Based on this observed seasonality, we grouped circulating strains collected during November-April under the winter season and those collected during May-October under the summer season.

We successfully sequenced 502 influenza A(H3N2) and 481 influenza B samples covering all influenza seasons in the study period, except in 2009, when influenza A(H1N1) pdm09 dominated activity. In the phylogenetic analysis based on HA sequences, H3N2 virus exhibited a characteristic ladder-like tree topology suggesting continuous 


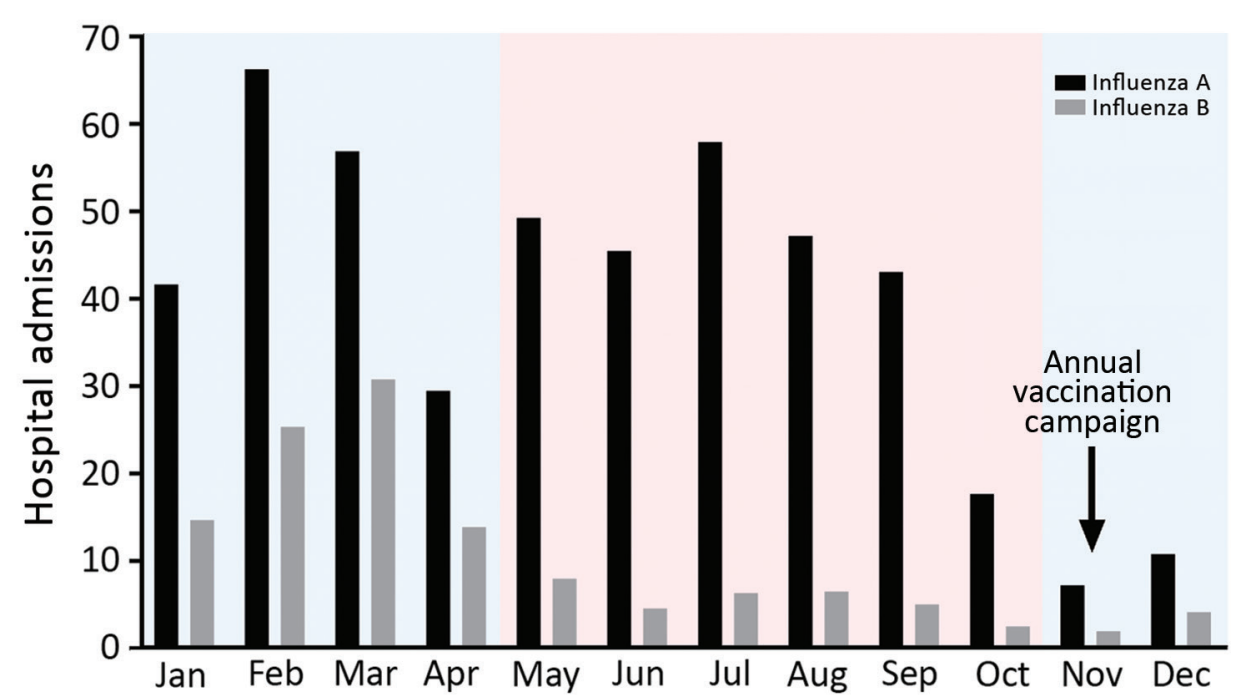

Figure 1. Seasonality of influenza A and B, Hong Kong, China, 1996-2012. The numbers of patients hospitalized with acute respiratory illnesses and who had laboratory-confirmed influenza were retrieved from a computerized laboratory information system at the microbiology department of a district general hospital that serves $9 \%$ (0.6 million) of Hong Kong's population. Pink indicates summer seasons; blue indicates winter seasons.

unidirectional evolution (Figure 2, panel A). Existing H3N2 variants circulated for a median of 2 years (range 1-4 years), with gaps between the winter and summer seasons, and became displaced rapidly by newly emerging variants. We observed a more complex tree topology for influenza B, where new lineages emerged from deep branches and evolved toward multiple directions (Figure 2 , panel B). In addition, multiple influenza B variants cocirculated for a median of 6 years (range 2-15 years) before being displaced by new variants.

Of the 983 circulating strains examined, 8 (maximum 2 per season) influenza $\mathrm{A}(\mathrm{H} 3 \mathrm{~N} 2$ ) and 12 (maximum 4 per season) influenza B viruses were regarded as far away from any vaccine strains recommended during the 17-year study period. The pairwise HA amino acid distances of these strains were more than twice the SD from the mean of the related clusters (online Technical Appendix Figures 1, 2, https://wwwnc.cdc.gov/EID/article/24/10/18-0652Techapp1.pdf), and they were distant from all vaccine strains based on the phylogenetic tree topology.

\section{Matching Between Circulating and Vaccine Strains}

\section{Influenza $\mathbf{A}(\mathrm{H} 3 \mathrm{~N} 2)$}

We assessed the temporal relationship between circulating $\mathrm{H} 3 \mathrm{~N} 2$ viruses collected in this study and their closest Northern Hemisphere vaccine strain based on HA amino acid sequence comparisons (Figure 3, panel A). Most Northern hemisphere vaccine-like strains (9/11) had circulated in Hong Kong for $>1$ year before WHO first recommended them for inclusion in the Northern Hemisphere vaccine. We found co-circulation of multiple strains in 17 $(50.0 \%)$ of the 34 seasons. In 13 (38.2\%) seasons, at least a portion of the circulating viruses were closely matched with the contemporary vaccine strain. However, in only 7
$(20.6 \%)$ seasons, the closely matched portion accounted for the $>50 \%$ of circulating viruses examined. A full match was achieved in only $2(5.9 \%)$ seasons. We determined the proportion of circulating viruses that were genetically closely matched with vaccine strains based on HA amino acid sequence comparison (Table), which should not be taken as vaccine effectiveness because cross-protection between mismatched strains can occur.

We repeated the same analysis with respect to Southern Hemisphere vaccine strains. Similarly, most (8/11) Southern Hemisphere H3N2 virus vaccine strains had circulated in Hong Kong for $>1$ year before WHO first recommended them for inclusion in the Southern Hemisphere vaccine. A similar picture on matching was revealed (Figure 3, panel B). In $10(29.4 \%)$ of the 34 seasons, at least a portion of the circulating viruses were closely matched with the contemporary vaccine strain. However, only in 5 (14.7\%) seasons did the matched portion account for the $>50 \%$ of circulating viruses examined.

\section{Influenza B}

We assessed the temporal relationship between circulating influenza B viruses and their closely matched Northern Hemisphere vaccine strains based on HA amino acid sequence comparisons (Figure 4, panel A). Most $(6 / 7)$ of the Northern Hemisphere influenza B vaccine-like circulating strains had been found in Hong Kong $>1$ year before WHO first recommended them as vaccine strains. Co-circulation of multiple strains was found in 26 $(76.5 \%)$ of the 34 seasons examined. In 15 (44.1\%) seasons, at least some of the circulating viruses were closely matched with the contemporary Northern Hemisphere vaccine strain. However, in only $5(14.7 \%)$ seasons did the matched portion account for the $>50 \%$ of circulating viruses examined. 
Analysis of Southern Hemisphere vaccine strains showed a similar result (Figure 4, panel B). Most (5/6) of the vaccine-like strains were found in Hong Kong $>1$ year before WHO first recommended them as vaccine strains. Again, we found matched strains in $14(41.2 \%)$ seasons, but in only $4(11.8 \%)$ seasons did the matched viruses account for the $>50 \%$ of viruses examined.

\section{Strain Drifting between Winter and Summer Seasons}

Influenza A(H3N2)

In 7 of the 17 years $(2002,2003,2004,2005,2007,2009$, and 2011), the predominant $\mathrm{H} 3 \mathrm{~N} 2$ virus strain in summer differed from that in the preceding winter. We further

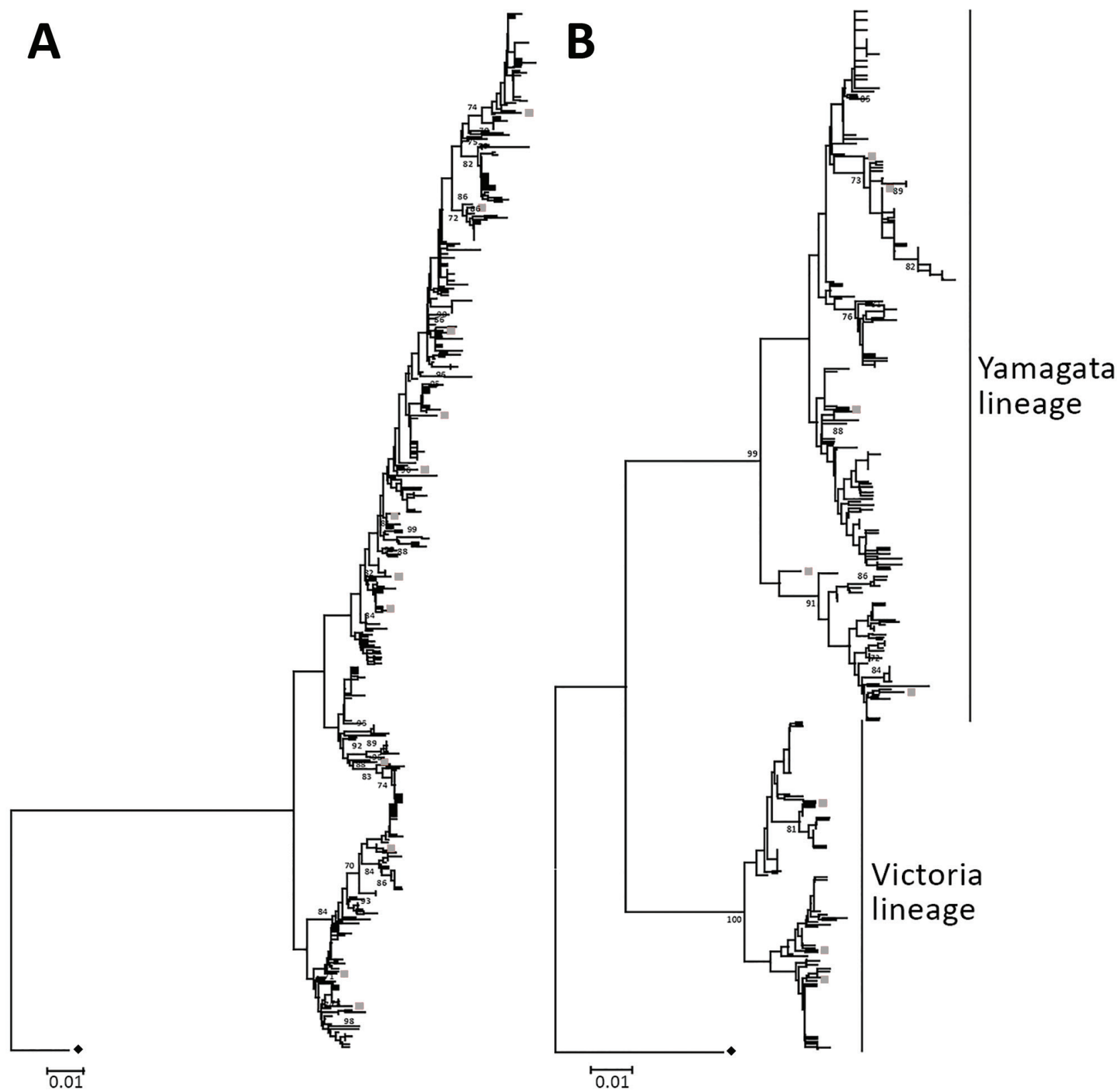

Figure 2. Neighbor-joining phylogenetic inference of near-complete hemagglutinin protein of influenza $A(H 3 N 2)(A)$ and influenza $B$ (B), Hong Kong, China, 1996-2012. Pairwise protein sequence distances were calculated using a Poisson model. Triangles denote ancestral influenza strains: influenza A(H3N2), A/Hong Kong/1/1968, GenBank accession no. CY044261; influenza B, B/Lee/1940, accession no. CY115111. Gray squares denote recommended vaccine strains; for clarity, only those used in Figures 3 and 4 are shown. Bootstrap values $>70 \%$ are shown at the respective nodes. All alignment positions containing gaps were omitted from the analysis. Trees were rooted to ancestral strains and drawn to scale. Scale bars indicate numbers of amino acid substitutions per position. GenBank accession numbers of hemagglutinin sequences of circulating strains collected in this study are shown in the online Technical Appendix Table (https://wwwnc.cdc.gov/EID/article/24/10/18-0652-Techapp1.pdf). 
examined whether administration of an additional Southern Hemisphere vaccine at the beginning of summer (around May) could achieve better vaccine matching. In 5 of the 7 years, content of newly available Southern Hemisphere vaccines was identical to the preceding Northern Hemisphere vaccine composition. In 2004 and 2005, although another strain was chosen for the Southern Hemisphere vaccine, the new vaccine strain was not closely matched with circulating viruses at that time. These results suggested that additional administration of Southern Hemisphere vaccines at the beginning of summer would not have improved the immunity against the circulating H3N2 strains.

\section{Influenza B}

In 6 of the 17 years $(2000,2002,2004,2008,2010$, and 2011), a drift occurred in the predominant influenza $B$ variant from winter to summer seasons. Similarly, we examined the value of an additional Southern Hemisphere vaccine administered at the beginning of summer and again found no benefit. In 5 of the 6 drift years, the newly recommended Southern Hemisphere vaccine composition was identical to the previous Northern Hemisphere vaccine. In 2008, although the Southern Hemisphere vaccine contained a new virus (Yamagata, B/Florida/4/2006) that closely matched the circulating viruses detected in the first half (winter season) of the same year, the circulating strain

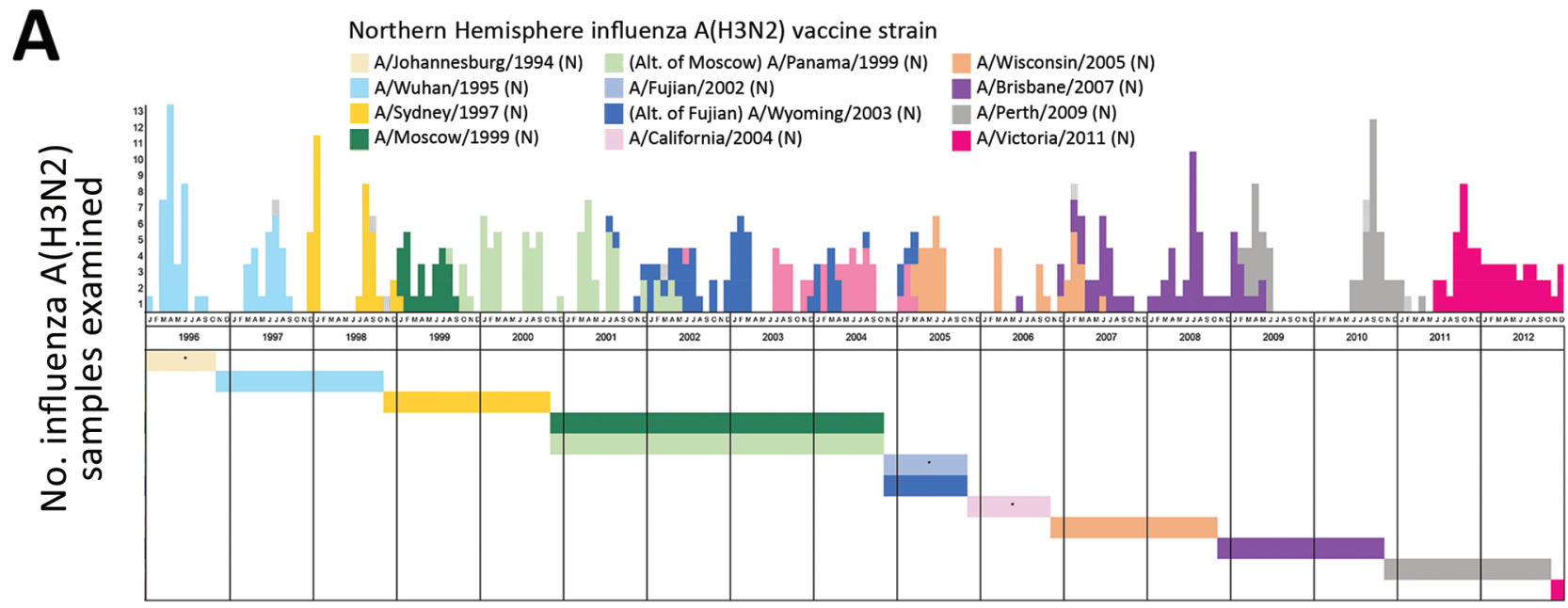

B

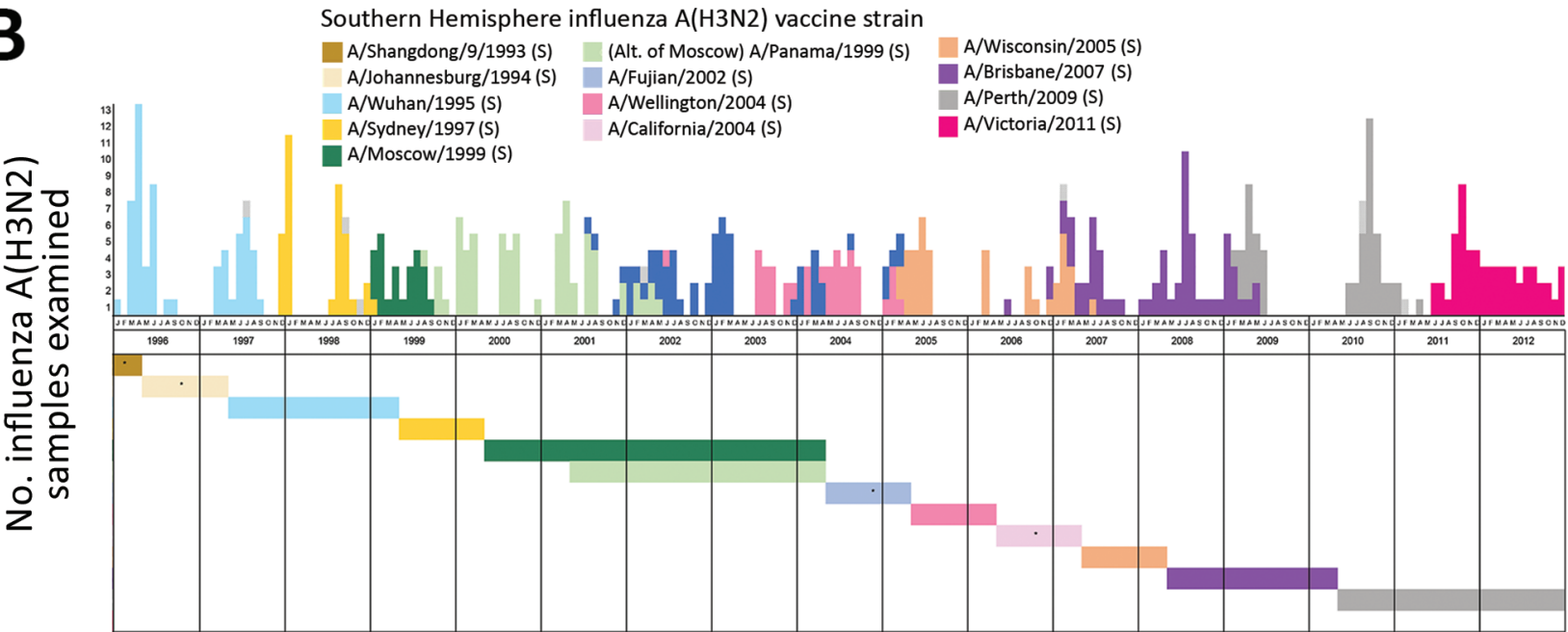

Figure 3. Matching between circulating and vaccine strains of influenza A(H3N2), Hong Kong, China, 1996-2012. Each circulating virus was assigned on the basis of full-length hemagglutinin amino acid distance and phylogenetic tree topology to the closest World Health Organization-recommended influenza A(H3N2) vaccine strain for Northern Hemisphere $(A)$ and Southern Hemisphere (B) vaccines. Closely matched viruses are labeled with the same color. The circulating strains with no closest vaccine strain identified as defined in the Methods are indicated by dotted boxes. Horizontal color bars indicate the protection period of the corresponding vaccine strain. Asterisks indicate vaccine strains without closely matched circulating viruses. The protection period of Northern Hemisphere vaccines was defined as from November through the following October and of Southern Hemisphere vaccines from May through the following April. All the alternative vaccine strains were analyzed, and those with different results are shown. 
Table. Proportion of circulating influenza viruses with HA amino acid sequences closely matched with vaccine strains, Hong Kong, China, 1996-2012*

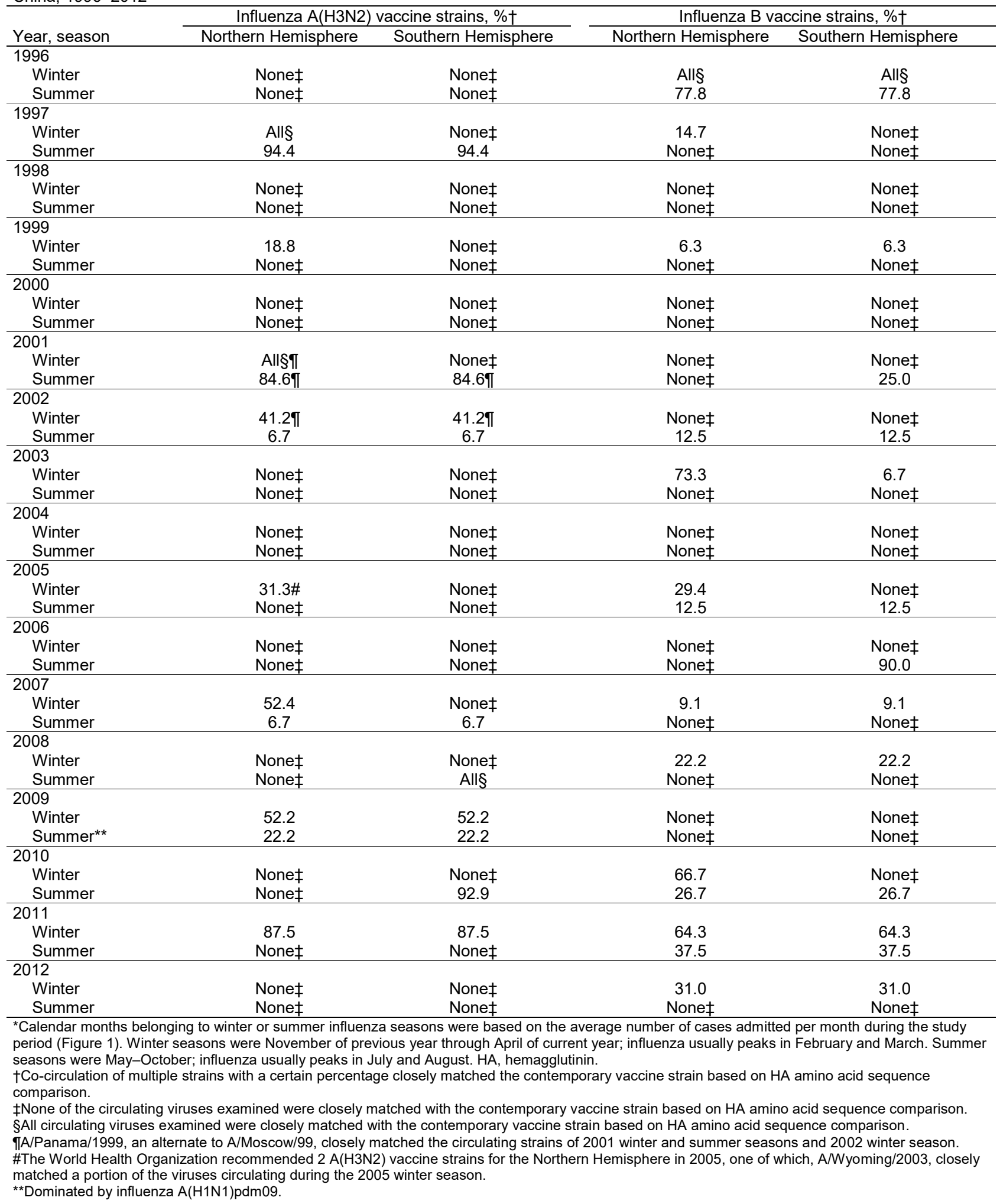


was soon replaced by another influenza B variant not covered in the recommended Southern Hemisphere vaccine.

\section{Discussion}

This study focused on the vaccine matching of influenza $\mathrm{A}(\mathrm{H} 3 \mathrm{~N} 2)$ and influenza $\mathrm{B}$ viruses because antigenic drift frequently occurs in these viruses, leading to changes in vaccine recommendation, more so than for influenza $\mathrm{A}(\mathrm{H} 1 \mathrm{~N} 1)$ virus (7). Antigenic characterization of influenza viruses, and thus the assessment of vaccine matching, has traditionally been based on hemagglutination inhibition (HI) assay. HI measures the ability of specific antibodies, typically reference ferret antiserum, to inhibit the binding of virus HA to receptors on erythrocytes. However, H3N2 virus variants from the past 3 decades have progressively displayed reduced avidity to erythrocytes of commonly used animal species, making the results of HI assays difficult to interpret $(8,18)$. In this study, we analyzed the amino acid sequence of HA to infer vaccine matching because genetic and antigenic characteristics of influenza viruses display remarkable correspondence, and both carry a strong correlation with vaccine effectiveness (19-21). The molecular phylogenetic approach used enables direct analyses of viruses in clinical samples rather than relying on cell culture-propagated isolates. Furthermore, the molecular approach does not require influenza variant-specific antiserum and is more feasible for large-scale epidemiologic surveys. One caution in interpreting molecular results is

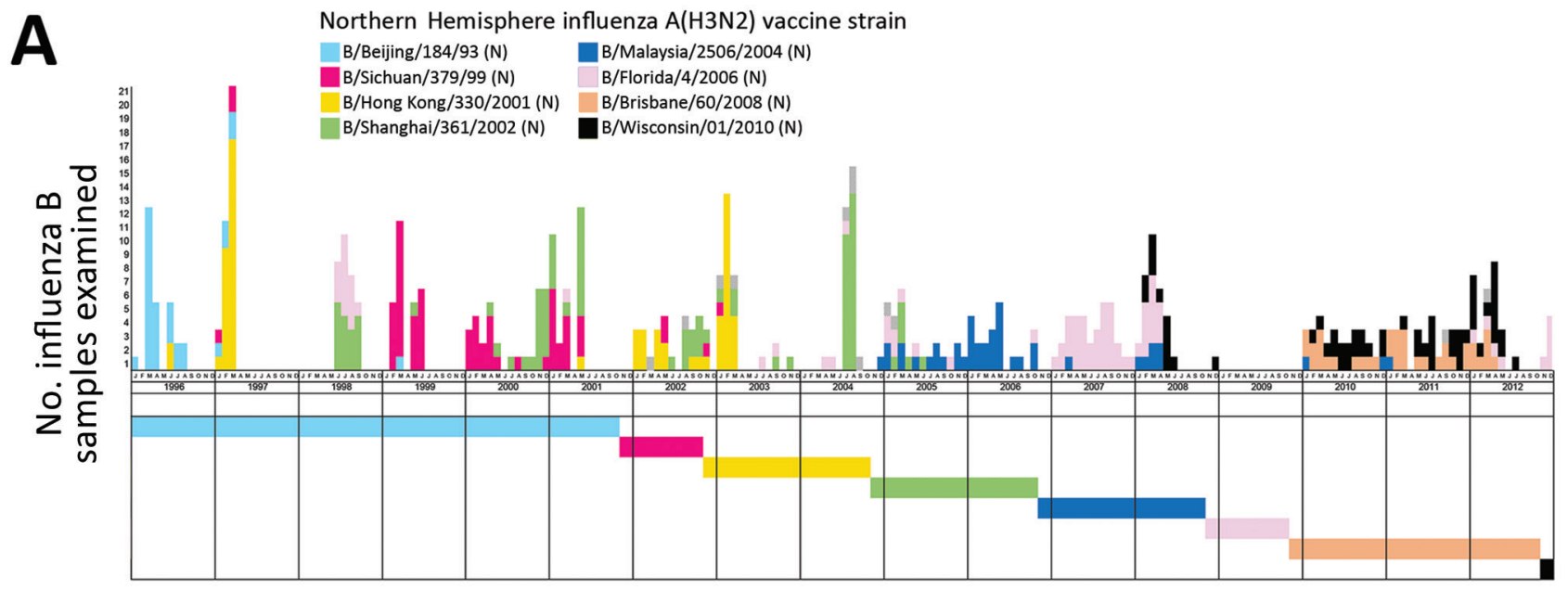

B

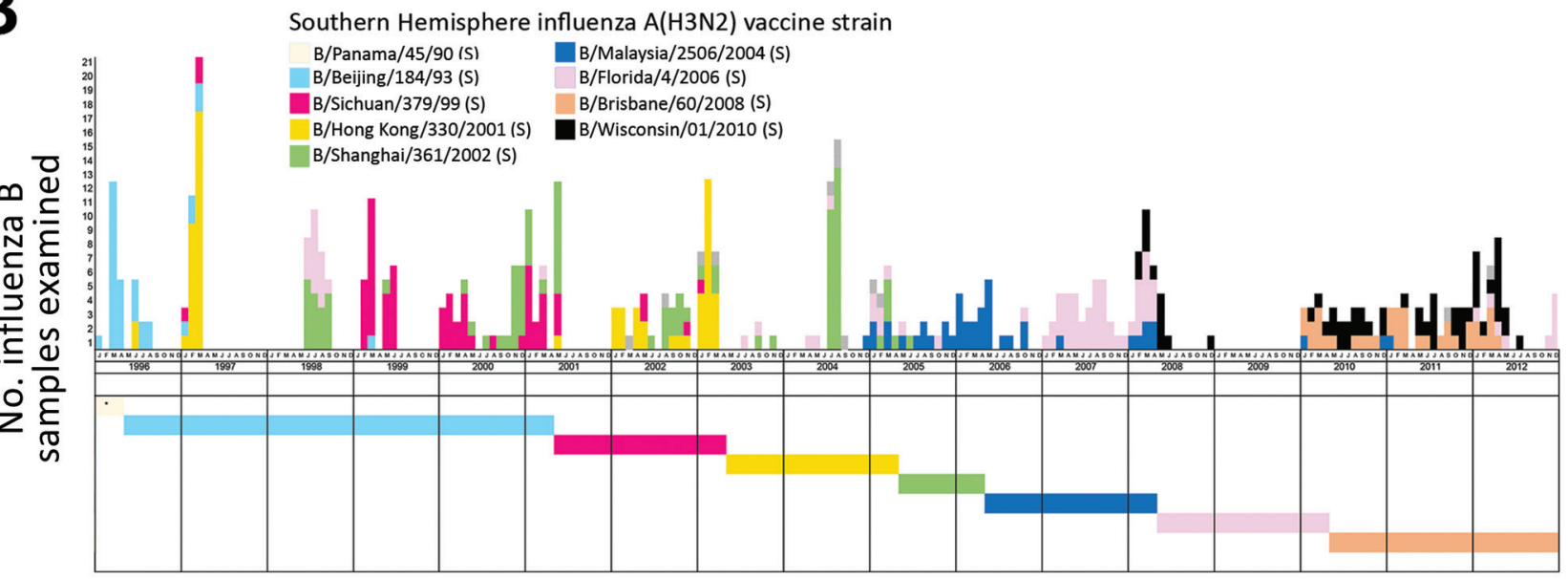

Figure 4. Matching between circulating and vaccine strains of influenza B, Hong Kong, China, 1996-2012. Each circulating virus was assigned on the basis of full-length hemagglutinin amino acid distances and phylogenetic tree topology to the closest World Health Organization-recommended influenza B vaccine strain for Northern Hemisphere $(A)$ and Southern Hemisphere (B) vaccines. Closely matched viruses are labeled with the same color. The circulating strains with no closest vaccine strain identified as defined in the Methods are indicated by dotted boxes. Horizontal color bars indicate the protection period of the corresponding vaccine strain. Asterisks indicate vaccine strains without closely matched circulating viruses. The protection period of Northern Hemisphere (A) vaccines was defined as from November through the following October and of Southern Hemisphere (B) vaccines from May through the following April. All the alternative vaccine strains were analyzed, and those with different results are shown. 
that influenza vaccines might still display cross-protection efficacy against circulating variants that are regarded as not closely matched to the vaccine strains based on genetic comparison. Nevertheless, our approach to define matching between vaccine and circulating strains would be sufficient to guide the selection of Northern and Southern Hemisphere vaccines and to inform the potential benefit of a biannual vaccination strategy using both Northern and Southern Hemisphere vaccines on the background of influenza epidemiology faced by subtropical countries.

Given the extensive amount of travel between Hong Kong and neighboring East Asia cities, we expect a substantial sharing of viruses detected in Hong Kong with the region. Although the proportion of infectious travelers is difficult to estimate, a study from Xiamen International Airport (Xiamen, China) in 2015-2016 found that $\approx 8 / 10,000$ incoming travelers had influenza-like illness, of whom $21 \%$ tested positive for influenza (22). The fact that Hong Kong shares a similar, though not always synchronized, pool of influenza viruses with the neighboring East and Southeast Asian cities is supported by a study from Bahl et al. (23) and sequence information from Nextstrain (https:// nextstrain.org). East and Southeast Asia are the sources of new genetic variants of H3N2 virus, which seed epidemics worldwide (24-28). In line with this, we demonstrated that most of the $\mathrm{H} 3 \mathrm{~N} 2$ virus strains selected for inclusion in influenza vaccines could be detected in East Asia $>1$ year before recommendation by WHO. Most of these newly emerged strains predominated for 2 years, covering 4 influenza seasons in this region. Although vaccine compositions were reviewed twice a year, when vaccines incorporating newly emerged strains were made available to the market, often another newly drifted influenza strain had become predominant in the region. Based on our analysis covering 17 years with 34 influenza seasons, the probability of a season having the contemporary vaccine closely matched with $>50 \%$ of the circulating viruses was only $20.6 \%$, and if we aimed at matching $>70 \%$ of circulating viruses, the probability was further reduced to $14.7 \%$. As reflected in a recent study on vaccine effectiveness against hospitalization among children in Hong Kong, efficacy against H3N2 virus was on average $36.6 \%$ (29).

Because Hong Kong, like many other subtropical cities, faces multiple influenza peaks annually, we raised the question whether Southern Hemisphere influenza vaccines would provide timely and better vaccine matching. However, we observed that most of the time composition of the Southern Hemisphere vaccine is the same as the preceding Northern Hemisphere vaccine and thus made no difference in vaccine matching. Furthermore, according to the current production schedule, Southern Hemisphere vaccines are made available in May, just after the period with highest influenza activity in Hong Kong (January-March).
Therefore, to administer influenza vaccine in May requires vaccines with efficacy lasting for almost 12 months, which is not a demonstrated property of current vaccines.

Given the comparable duration of winter and summer influenza seasons in Hong Kong, the next question was whether biannual vaccination with Northern and Southern Hemisphere vaccines administered before the winter and summer seasons, respectively, would improve immunity against circulating strains. In early 2015, Hong Kong faced a strong winter influenza peak associated with a newly drifted variant, influenza $\mathrm{A}(\mathrm{H} 3 \mathrm{~N} 2) / \mathrm{Sw}$ itzerland, which was not incorporated into the Northern Hemisphere vaccine. In view of a possible strong summer peak, an ad hoc vaccination campaign was conducted in May using the newly available Southern Hemisphere vaccine that incorporated the new variant. However, willingness to accept the vaccine was low among healthcare professionals, and a major proportion expressed concern (30). Furthermore, in recent years, concern has surfaced that repeated influenza vaccination might negatively affect current vaccine effectiveness (31-33). Although a recent meta-analysis does not support such a claim (34), this possibility should not be ignored when considering biannual vaccination. Although the cost-effectiveness and feasibility of a biannual vaccination strategy is yet to be assessed, our analysis does not support that an additional vaccine before summer seasons can improve matching. We observed a substantial chance of drift in predominant influenza viruses from season to season $(41.2 \%$ for $\mathrm{H} 3 \mathrm{~N} 2$ and $35.3 \%$ for influenza B), and the newly available Southern Hemisphere vaccines typically had the same strain composition as the preceding Northern Hemisphere vaccine or the updated Southern Hemisphere composition still did not achieve a close match with the then-circulating viruses.

East Asia and other subtropical regions face different challenges in achieving close vaccine matching for influenza $\mathrm{A}(\mathrm{H} 3 \mathrm{~N} 2)$ and influenza $\mathrm{B}$. For H3N2 virus, the forthcoming predominant strain can be identified from a subtropical region-based surveillance network. The newly emerged strains usually predominate for 1-2 years, which cover 4 influenza seasons in this region. If recommendation on vaccine composition followed by manufacturing is accomplished within a few months, a close match can be achieved in the subsequent 2-3 seasons to provide the best possible vaccine effectiveness. This streamlined workflow should be practicable with current logistics and production provided that a sustainable market can be established in the region.

The challenge posed by influenza B is completely different. Although Bedford et al. suggested that East and Southeast Asia play limited roles in disseminating new variants of influenza B (24), we observed that most of the influenza B strains could be found in East Asia long 
before they are selected as vaccine strains. East Asia is therefore also an important site for identifying emergent influenza B strains. However, co-circulation and multiple reintroductions of existing and emerging influenza strains complicate selection of vaccine strains. Incorporating both lineages of influenza B into the quadrivalent vaccine might be able to solve at least part of the problem. In either case, a next-generation influenza vaccine should be designed to provide broad cross-protection against different antigenic variants (35).

East Asia is an important contributor to the influenza virus surveillance program. However, vaccine strains incorporated in the Northern and Southern Hemisphere influenza vaccines frequently do not match the contemporary circulating viruses in this region. Even biannual vaccination is unlikely to improve vaccine matching. A specific strategy is urgently needed to select and produce influenza vaccines targeting the tropical and subtropical regions.

This work was supported by Area of Excellence Scheme, University Grants Committee, Hong Kong [AoE/M-12/06].

\section{About the Author}

Dr. Martin C.W. Chan is an assistant professor in the Department of Microbiology of the Chinese University of Hong Kong. His primary research interests are molecular epidemiology of acute viral infections, especially those caused by influenza viruses and noroviruses; use of metagenomic next-generation sequencing in clinical diagnostics; gut virome; and food virology.

\section{References}

1. Biggerstaff M, Cauchemez S, Reed C, Gambhir M, Finelli L. Estimates of the reproduction number for seasonal, pandemic, and zoonotic influenza: a systematic review of the literature. BMC Infect Dis. 2014;14:480. http://dx.doi.org/10.1186/ 1471-2334-14-480

2. World Health Organization. Influenza (seasonal). Fact sheet. 2016 [cited 2017 Jun 15]. http://www.who.int/mediacentre/factsheets/ fs211/en/

3. Bennett JE, Dolin R, Blaser MJ. Mandell, Douglas, and Bennett's principles and practices of infectious diseases, 8 th ed. Philadelphia: Elsevier; 2015.

4. Cox NJ, Subbarao K. Global epidemiology of influenza: past and present. Annu Rev Med. 2000;51:407-21. http://dx.doi.org/ 10.1146/annurev.med.51.1.407

5. Carrat F, Flahault A. Influenza vaccine: the challenge of antigenic drift. Vaccine. 2007;25:6852-62. http://dx.doi.org/10.1016/ j.vaccine.2007.07.027

6. Houser K, Subbarao K. Influenza vaccines: challenges and solutions. Cell Host Microbe. 2015;17:295-300. http://dx.doi.org/ 10.1016/j.chom.2015.02.012

7. World Health Organization. WHO recommendations on the composition of influenza virus vaccines [cited 2017 Aug 10]. http://www.who.int/influenza/vaccines/virus/recommendations/en/

8. Hampson A, Barr I, Cox N, Donis RO, Siddhivinayak H, Jernigan D, et al. Improving the selection and development of influenza vaccine viruses - report of a WHO informal consultation on improving influenza vaccine virus selection, Hong Kong SAR,
China, 18-20 November 2015. Vaccine. 2017;35:1104-9. http://dx.doi.org/10.1016/j.vaccine.2017.01.018

9. Kong H. Hong Kong: the facts [cited 2017 Sep 6]. https://www.gov.hk/en/about/abouthk/factsheets/docs/population.pdf

10. Census and Statistics Department, Government of the Hong Kong Special Administrative Region. Transport, communication and tourism. Cross-boundary travel survey 2015 [cited 2018 Jun 7]. https://www.censtatd.gov.hk/hkstat/sub/sp130. jsp?productCode $=$ FA100267

11. Census and Statistics Department, Government of the Hong Kong Special Administrative Region. Transport, communication and tourism. Table E551: visitor arrivals by country/region of residence [cited 2018 Jun 7]. https://www.censtatd.gov.hk/hkstat/sub/sp130. jsp?productCode $=$ D5600551

12. Chan PK, Tam WW, Lee TC, Hon KL, Lee N, Chan MC, et al. Hospitalization incidence, mortality, and seasonality of common respiratory viruses over a period of 15 years in a developed subtropical city. Medicine (Baltimore). 2015;94:e2024. http://dx.doi.org/10.1097/MD.0000000000002024

13. Tang JW, Ngai KL, Lam WY, Chan PK. Seasonality of influenza A(H3N2) virus: a Hong Kong perspective (1997-2006). PLoS One. 2008;3:e2768. http://dx.doi.org/10.1371/journal.pone.0002768

14. World Health Organization. Influenza Research Database. World Health Organization recommendations for composition of influenza vaccines [cited 2017 Aug 10]. https://www.fludb.org/brc/ vaccineRecommend.spg?decorator=influenza

15. Tamura K, Stecher G, Peterson D, Filipski A, Kumar S. MEGA6: molecular evolutionary genetics analysis version 6.0. Mol Biol Evol. 2013;30:2725-9. http://dx.doi.org/10.1093/molbev/mst197

16. Zuckerkandl E, Pauling L. Evolutionary divergence and convergence in proteins. In: Bryson V, Vogel HJ, editors. Evolving genes and proteins. New York: Academic Press; 1965. p. 97-166.

17. Shu YL, Fang LQ, de Vlas SJ, Gao Y, Richardus JH, Cao WC. Dual seasonal patterns for influenza, China. Emerg Infect Dis. 2010;16:725-6. http://dx.doi.org/10.3201/eid1604.091578

18. Lin YP, Xiong X, Wharton SA, Martin SR, Coombs PJ, Vachieri SG, et al. Evolution of the receptor binding properties of the influenza A(H3N2) hemagglutinin. [Erratum in: Proc Natl Acad Sci U S A. 2013;110:2677. http://dx.doi.org/10.1073/ pnas.1218841110]. Proc Natl Acad Sci U S A. 2012;109:21474-9.

19. Smith DJ, Lapedes AS, de Jong JC, Bestebroer TM, Rimmelzwaan GF, Osterhaus AD, et al. Mapping the antigenic and genetic evolution of influenza virus. Science. 2004;305:371-6. http://dx.doi.org/10.1126/science.1097211

20. Lee MS, Chen JS. Predicting antigenic variants of influenza A/ H3N2 viruses. Emerg Infect Dis. 2004;10:1385-90. http://dx.doi.org/10.3201/eid1008.040107

21. Gupta V, Earl DJ, Deem MW. Quantifying influenza vaccine efficacy and antigenic distance. Vaccine. 2006;24:3881-8. http://dx.doi.org/10.1016/j.vaccine.2006.01.010

22. Chen J, Yang K, Zhang M, Shen C, Chen J, Wang G, et al. Rapid identification of imported influenza viruses at Xiamen International Airport via an active surveillance program. Clin Microbiol Infect. 2018;24:289-94. http://dx.doi.org/10.1016/j.cmi.2017.05.027

23. Bahl J, Nelson MI, Chan KH, Chen R, Vijaykrishna D, Halpin RA, et al. Temporally structured metapopulation dynamics and persistence of influenza A H3N2 virus in humans. Proc Natl Acad Sci U S A. 2011;108:19359-64. http://dx.doi.org/10.1073/pnas.1109314108

24. Bedford T, Riley S, Barr IG, Broor S, Chadha M, Cox NJ, et al. Global circulation patterns of seasonal influenza viruses vary with antigenic drift. Nature. 2015;523:217-20. http://dx.doi.org/ 10.1038/nature 14460

25. Russell CA, Jones TC, Barr IG, Cox NJ, Garten RJ, Gregory V, et al. The global circulation of seasonal influenza A (H3N2) viruses. Science. 2008;320:340-6. http://dx.doi.org/10.1126/ science. 1154137 
26. Lemey P, Rambaut A, Bedford T, Faria N, Bielejec F, Baele G, et al. Unifying viral genetics and human transportation data to predict the global transmission dynamics of human influenza H3N2. PLoS Pathog. 2014;10:e1003932. http://dx.doi.org/10.1371/ journal.ppat. 1003932

27. Bedford T, Cobey S, Beerli P, Pascual M. Global migration dynamics underlie evolution and persistence of human influenza A (H3N2). PLoS Pathog. 2010;6:e1000918. http://dx.doi.org/10.1371/ journal.ppat.1000918

28. Chan J, Holmes A, Rabadan R. Network analysis of global influenza spread. PLOS Comput Biol. 2010;6:e1001005. http://dx.doi.org/10.1371/journal.pcbi.1001005

29. Cowling BJ, Chan KH, Feng S, Chan EL, Lo JY, Peiris JS, et al. The effectiveness of influenza vaccination in preventing hospitalizations in children in Hong Kong, 2009-2013. Vaccine. 2014;32:5278-84. http://dx.doi.org/10.1016/j.vaccine.2014.07.084

30. Wong MC, Nelson EA, Leung C, Lee N, Chan MC, Choi KW, et al Ad hoc influenza vaccination during years of significant antigenic drift in a tropical city with 2 seasonal peaks: a cross-sectional survey among health care practitioners. Medicine (Baltimore). 2016;95:e3359. http://dx.doi.org/10.1097/MD.0000000000003359

31. Tam YH, Valkenburg SA, Perera RAPM, Wong JHF, Fang VJ, $\mathrm{Ng}$ TWY, et al. Immune responses to twice-annual influenza vaccination in older adults in Hong Kong. Clin Infect Dis. 2018;66:904-12. http://dx.doi.org/10.1093/cid/cix900
32. McLean HQ, Thompson MG, Sundaram ME, Meece JK, McClure DL, Friedrich TC, et al. Impact of repeated vaccination on vaccine effectiveness against influenza $\mathrm{A}(\mathrm{H} 3 \mathrm{~N} 2)$ and $\mathrm{B}$ during 8 seasons. Clin Infect Dis. 2014;59:1375-85. http://dx.doi.org/ 10.1093/cid/ciu680

33. Skowronski DM, Chambers C, Sabaiduc S, De Serres G, Winter AL, Dickinson JA, et al. A perfect storm: impact of genomic variation and serial vaccination on low influenza vaccine effectiveness during the 2014-2015 season. Clin Infect Dis. 2016;63:21-32. http://dx.doi.org/10.1093/cid/ciw176

34. Ramsay LC, Buchan SA, Stirling RG, Cowling BJ, Feng S, Kwong JC, et al. The impact of repeated vaccination on influenza vaccine effectiveness: a systematic review and meta-analysis. BMC Med. 2017;15:159. http://dx.doi.org/10.1186/ s12916-017-0919-0

35. Pica N, Palese P. Toward a universal influenza virus vaccine: prospects and challenges. Annu Rev Med. 2013;64:189-202. http://dx.doi.org/10.1146/annurev-med-120611-145115

Address for correspondence: Paul K.S. Chan, The Chinese University of Hong Kong, Department of Microbiology, 1/F LCW Clinical Sciences Bldg., Prince of Wales Hospital,, Shatin, New Territories, Hong Kong Special Administrative Region, China; email: paulkschan@cuhk.edu.hk

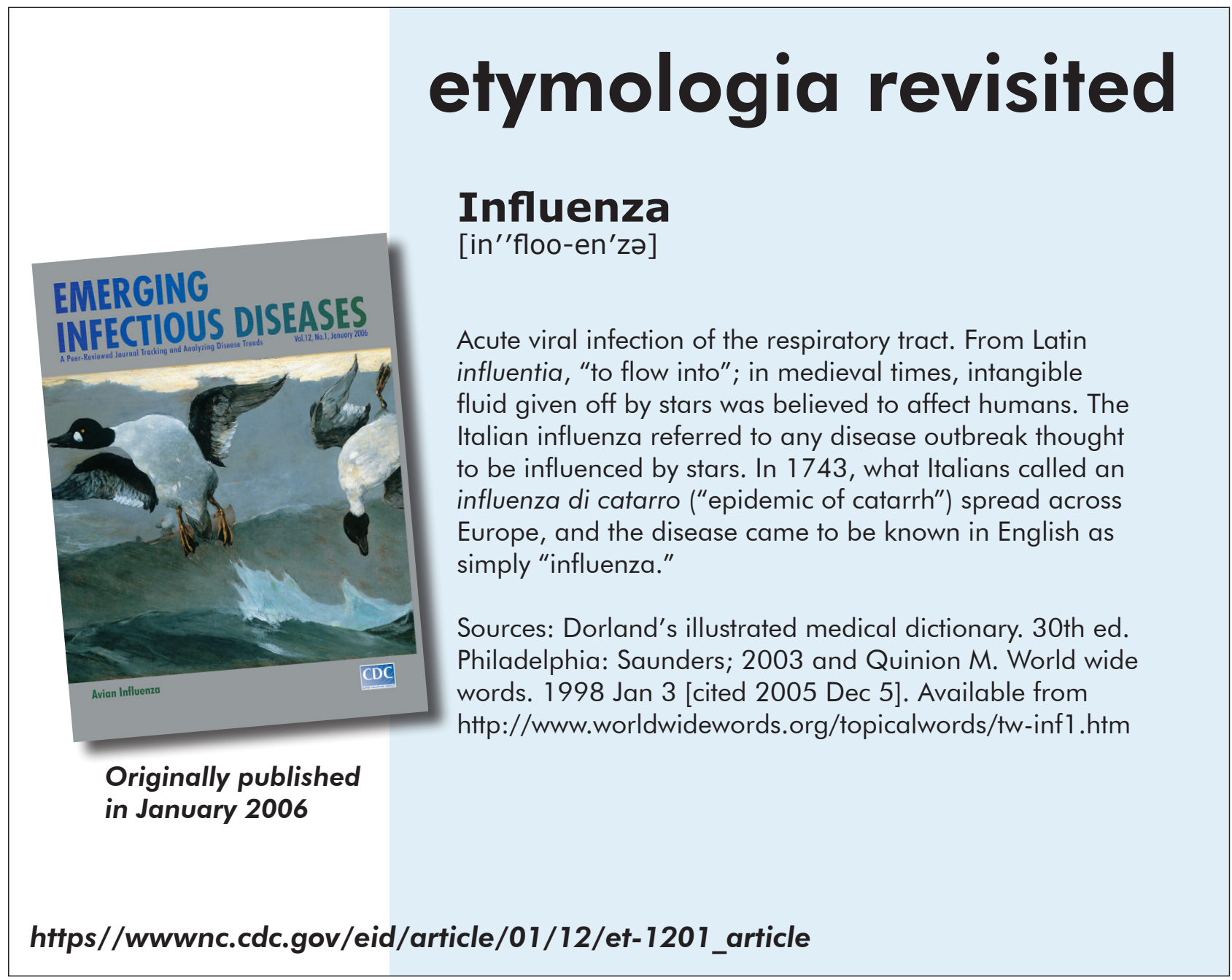

